\title{
Article
}

\section{Designing Sensing Devices Using Porous Composite Materials}

\author{
Chang-Ming Wang and Wei-Ssu Liao *(D) \\ Department of Chemistry, National Taiwan University, Taipei 10617, Taiwan; cmwangzc@ntu.edu.tw \\ * Correspondence: wsliaochem@ntu.edu.tw
}

check for

updates

Citation: Wang, C.-M.; Liao, W.-S. Designing Sensing Devices Using Porous Composite Materials. J. Compos. Sci. 2021, 5, 35. https:// doi.org/10.3390/jcs5010035

Received: 25 December 2020

Accepted: 15 January 2021

Published: 19 January 2021

Publisher's Note: MDPI stays neutral with regard to jurisdictional claims in published maps and institutional affiliations.

Copyright: (c) 2021 by the authors. Licensee MDPI, Basel, Switzerland. This article is an open access article distributed under the terms and conditions of the Creative Commons Attribution (CC BY) license (https:// creativecommons.org/licenses/by/ $4.0 /)$.

\begin{abstract}
The need for portable and inexpensive analytical devices for various critical issues has led researchers to seek novel materials to construct them. Soft porous materials, such as paper and sponges, are ideal candidates for fabricating such devices due to their light weight and high availability. More importantly, their great compatibility toward modifications and add-ons allows them to be customized to match different objectives. As a result, porous material-based composites have been extensively used to construct sensing devices applied in various fields, such as point-of-care testing, environmental sensing, and human motion detection. In this article, we present fundamental thoughts on how to design a sensing device based on these interesting composite materials and provide correlated examples for reader's references. First, a rundown of devices made with porous composite materials starting from their fabrication techniques and compatible detection methods is given. Thereafter, illustrations are provided on how device function and property improvements are achieved with a delicate use of composite materials. This includes extending device lifetime by using polymer films to protect the base material, while signal readout can be enhanced by a careful selection of protective cover and the application of advanced photo image analysis techniques. In addition to chemical sensors, mechanical responsive devices based on conductive composite materials are also discussed with a focus on base material selection and platform design. We hope the ideas and discussions presented in this article can help researchers interested in designing sensing devices understand the importance and usefulness of composite materials.
\end{abstract}

Keywords: porous material; sensor; composite material; analysis; device

\section{Porous Material-Based Composites for Sensing Devices}

The importance of inexpensive and portable analytical devices is on the rise due to critical issues such as point-of-care application, chemical and biological detection, and environmental pollutant sensing. Practical design and straightforward fabrication of such devices often require composites of multiple distinct materials and methods, and the integration of diverse science and engineering disciplines. Porous material-based composites, which are obtained by physical or chemical modification of native porous materials, retain the base materials' favorable physical properties. They are ideal for portable sensing devices because of their low weight, while the rendering large surface area allows efficient chemical processing. In addition, these materials are readily available and highly compatible with various sensing mechanisms [1-3]. These characteristics have led to their extensive use in constructing sensing devices for different research fields. For example, a porous paper material-based microfluidic chip designed by Reboud et al. delivers the full DNA diagnostics of malaria in blood samples, including sample preparation from whole-blood, isothermal amplification, and visualization of the output properties [4]. In another design proposed by Hiraoka et al., a distance-based sensor using composites of porous paper and a combination of chrome azurol S, lead chloride, tetrabromophenol blue, and citric buffer allows an instrument-free naked-eye evaluation of human urinary albumin/creatinine ratio in $15 \mathrm{~min}$ [5]. For environmental pollutant sensing, a Janus electrochemical device was constructed by printing wax-defined channels on porous paper, 
which can combine with various chemical reagents for simultaneous detection of $\mathrm{Cd}(\mathrm{II})$, $\mathrm{Pb}(\mathrm{II}), \mathrm{Cu}(\mathrm{II}), \mathrm{Fe}(\mathrm{II})$, and $\mathrm{Ni}(\mathrm{II})$ ions [6].

As demonstrated in these sensing devices, porous material-based composites are incredibly useful and potent when an analytical platform is designed. Although diverse device types and sensing target categories can be offered by different composite models, the platform construction concept and important performance affecting parameters are similar when working in this research field. To summarize recent works and for the ease of readers interested in these topics, we outline the underlying concepts, including material selection, device design, performance optimization, and signal output enhancement, in preparing a sensor based on porous material composites (Figure 1). Rather than giving a comprehensive review on all devices based on porous composite materials, this article focuses on bridging the aforementioned ideas towards practicality. Porous paper, a vastly popular selection for many sensing devices, is utilized as an example for interpretation. Beyond the illustration of platform design guidelines, other representative examples are also included to demonstrate the plausible expansion of these ideas and extensive correlated application fields (Table 1). At the end of this article, a discussion of future challenges and perspective opportunities are provided, which should be helpful for researchers planning to work on these exciting composite materials.

Table 1. Representative examples of sensing devices that utilize porous composite materials.

\begin{tabular}{|c|c|c|c|}
\hline Porous Composite & Target & Detection Method & Sensor Performance \\
\hline Filter paper/OPP-PVB/calcein blue & \multirow{2}{*}{ Metal ions } & Fluorometric & $\mathrm{Cu}^{2+}$ LOD: $1.28 \mu \mathrm{M}[7]$ \\
\hline Filter paper/PLA/eriochrome black $\mathrm{T}$ & & Colorimetric & $\mathrm{Ca}^{2+}$ and $\mathrm{Mg}^{2+}$ LOD: $0.08 \mathrm{mg} / \mathrm{L}$ [8] \\
\hline Filter paper/PET/Cu nanocluster & \multirow{5}{*}{$\begin{array}{l}\text { Small } \\
\text { molecules }\end{array}$} & Fluorometric & Cysteine LOD: $70 \mu \mathrm{M}$ [9] \\
\hline Chromatography paper/PET/HRP \& GOx & & Electrochemical & Glucose LOD: $16.2 \mu \mathrm{M}[10]$ \\
\hline Chromatography paper/wax/BuchE & & Electrochemical & $\begin{array}{l}\text { Methyl parathion LOD: } 0.06 \mathrm{nM} \\
{[11]}\end{array}$ \\
\hline Chromatography paper/wax/Cu NP & & Electrochemical & NOx LOD: 0.23 vppm [12] \\
\hline Filter paper/PLA/4-aminoantipyrane & & Colorimetric & Catechol LOD: $124 \mu \mathrm{g} / \mathrm{L}$ [8] \\
\hline $\begin{array}{c}\text { Chromatography paper/wax/Ag NP \& } \\
\text { antibodies }\end{array}$ & \multirow{2}{*}{ Biomolecules } & Electrochemical & Ricin LOD: 34 pM [13] \\
\hline $\begin{array}{l}\text { Chromatography paper/wax/Ag NP \& } \\
\text { DNA \& magnetic microbeads }\end{array}$ & & Electrochemical & HBV DNA LOD: 85 pM [14] \\
\hline $\begin{array}{c}\text { Chromatography paper/wax/AuPd NP \& } \\
\text { concanavalin A }\end{array}$ & \multirow[t]{2}{*}{ Cells } & Electrochemiluminescence & MCF-7 cells LOD: 250 cells/mL [15] \\
\hline Chromatography paper/wax/DNAzymes & & Colorimetric & E. coli K12 LOD: $10^{3}$ CFU/mL [16] \\
\hline Filter paper/PET/Ag NP-PEDOS:PSS & Pressure & Piezoresistive & Max sensitivity: $0.119 \mathrm{kPa}^{-1}[17]$ \\
\hline Polyurethane sponge/Ni(OH) 2 & \multirow{3}{*}{$\begin{array}{l}\text { Small } \\
\text { molecules }\end{array}$} & Electrochemical & Glucose LOD: $0.32 \mu \mathrm{M}[18]$ \\
\hline $\begin{array}{l}\text { PDMS-PU sponge } / \mathrm{InOH}_{3} \& \text { bromocresol } \\
\text { purple }\end{array}$ & & Colorimetric & Ammonia LOD: 50 ppb [19] \\
\hline Melamine sponge/graphene oxide & & Electrical & Ethanol LOD: 70 ppm [20] \\
\hline $\begin{array}{l}\text { PU foam/multiwalled carbon } \\
\text { nanotube-reduced graphene oxide }\end{array}$ & \multirow{3}{*}{ Pressure } & Piezoresistive & Max sensitivity: $0.088 \mathrm{kPa}^{-1}[21]$ \\
\hline $\begin{array}{l}\text { Melamine-formaldehyde sponge } / \mathrm{WS}_{2} \\
\text { nanosheet \& hydrophobic nanoparticles }\end{array}$ & & Piezoresistive & Max sensitivity: $0.39 \mathrm{kPa}^{-1}$ [22] \\
\hline PDMS/fragmented carbonized melamine & & Piezoresistive & Max gauge factor: 18.7 [23] \\
\hline
\end{tabular}




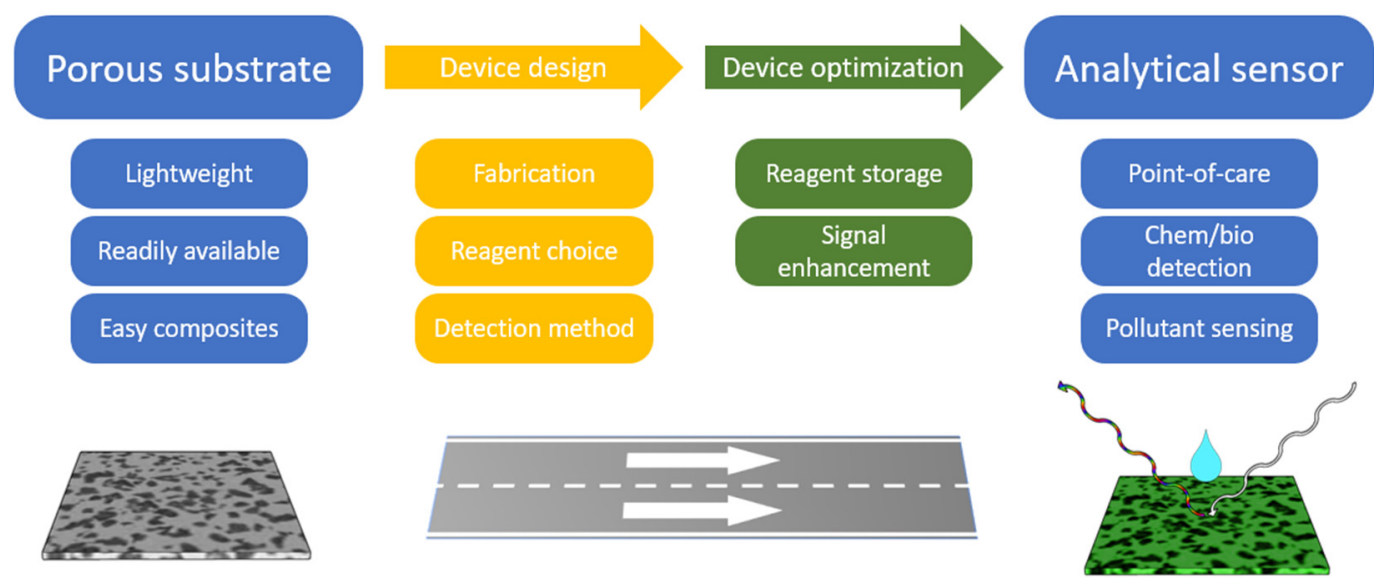

Figure 1. Key benefits of porous materials and considerations for their use in constructing sensing devices.

\section{Material Selection and Device Design}

In the construction of an analytical device, platform-supporting material selection and multiple functionality integration are crucial parameters to be considered. The determination of single or composite material usage relies on the purposes of an analytical device, and importantly, the design concepts of the produced platform. For instance, cellulose-based paper substrates have a long history of adoption for portable sensing purposes, from conventional pregnancy test strips to the recent developments popularized by Whitesides et al. [24]. This is due to advantageous characteristics of the soft porous paper material, as it has unparalleled compatibility with different fabrication techniques, reagent types, and detection methods. Besides, paper-based substrates are also intrinsically hydrophilic, which allows for the easy integration with aqueous-based chemical reactions and the spontaneous capillary wicking upon solution introduction. The capillary force that spontaneously propels aqueous solution through the substrate eliminates the need for an external driving source, and is critical when designing a miniature device for standalone and single uses. Great advancements of paper-based devices in the recent years can therefore be observed, ranging from developing new fabrication methods and novel reagents to inventing various fluid handling units. As can be seen in the representative example described above, the determination of suitable platform material for desired reagents and targets is the first task in an analytical device fabrication, where an optimal device design for reagent storage and performing analysis is also crucial.

In the design and fabrication of sensing devices, researchers can always take advantage of the supporting material properties not only in functionalities rendered by the device, but also the integration strategy that can be applied in the process. For example, soft porous paper materials' machinability implies that channels and reservoirs can be defined through subtractive shaping with ease, which is often accomplished by computer-aided designing and a plotter [25]. For these devices, the use of composite materials is common, and is often performed in conjunction with supporting polymer backings to increase device robustness. On the other hand, the porosity of the paper substrate allows the material hydrophilicity to be modified through impregnation of hydrophobic substances, such as photoresist [24], wax [26-28], and polydimethylsiloxane [29]. These hydrophobic chemicals can penetrate into the paper substrate pores, rendering the affected areas hydrophobic. Through appropriate designs of the introduced patterns, complex channels with various functionalities on a device can be realized. Among these reported strategies, the application of wax materials through a printing process is favored due to its relatively economical pricing, low toxicity, and simple operation. Furthermore, three-dimensional devices can also be obtained by stacking or folding multiple layers of paper substrates, allowing more functionality to be compiled into smaller devices [26,30]. 
When designing a portable sensing device, one should focus on minimizing the requirement of specialized equipment and well-trained operators. To this end, detection methods that utilize inexpensive and nontoxic reagents for in situ, real-time, and on-site data interpretation are preferred. Consequentially, colorimetric [8,9], fluorometric $[7,31,32]$, and electrochemical $[10,33]$ approaches are the most prominent on portable analytical devices. In colorimetric detections, a change in visual color after the introduction of analyte solution indicates the existence of targets, while the extent of color changes can often be used for rough quantification. Although the color change is ideally distinguishable with naked eyes, instrumental aids such as digital analysis can always help to achieve better quantification. Unlike colorimetric detections that produce changes in the visible light region of the spectrum, fluorometric detections rely on observing the alteration in fluorescence emission intensity under ultraviolet illumination. Despite issues such as background signal from additives in commercially available materials and more instrument/specialized experimental setting requirements, fluorescence sensing expands the arsenal of these devices, and has been utilized for the detection of small organic molecules, metal ions, bacteria, etc. By providing advantages such as higher sensitivity, better selectivity, and more stable data readout, the incorporation of electrochemical detection into porous substrate-based portable analytical devices has become increasingly popular. Various electrochemical sensing techniques, e.g., amperometry, voltammetry, potentiometry, have been demonstrated [34,35], and the detection of a wide array of targets, such as metal ions, small organic molecules, and $\mathrm{pH}$ value, has been achieved.

In short, various sensing devices can be fabricated using porous composite materials obtained by properly modifying porous materials with appropriate chemicals and processes. This step could affect the performance of a produced device, and the decision relies on specific aims and special requirements of the expected design.

\section{Device Performance Optimization}

Porous substrates allow easy storage and transportation of materials, but without proper handling, stored reagents and flowing analytes inside open pore substrates are at risk of environment contamination or interference during operation. Utilizing concepts similar to commercial packaging, lamination of a substrate with polymer films proves to be effective in improving handling experience. Such benefit was first emphasized by Cassano et al. [36], Liu et al. [37], de Oliveira et al. [38], and Wang et al. [10]. In addition to better handling brought forth by mechanical strength of the lamination film, a major advantage of enclosing a substrate with polymer films is the increased protection offered by the film. Environmentally sensitive reagents, such as enzymes or metal nanoclusters, are common picks as reagents for assays in porous substrate-based devices due to their high detection specificity and preparation customizability. These materials often experience reactivity loss over time as they spontaneously degrade. This drawback can be mitigated by sealing the reagent along with the porous substrate inside polymer films. Wang et al. demonstrated this idea by using a glucose-detecting system composed of potassium iodide, enzymatic glucose oxidase, and horseradish peroxidase [7]. The paper substrate was first roll-laminated into two pieces of polypropylene/polyvinyl butyral (OPP/PVB) composite film, and the reagents were thereafter spotted onto the paper substrate (Figure 2A). Device inlets were closed with a piece of tape to further reduce exposure of contents to the environment. The platform's responses to glucose were compared to unlaminated substrate pieces after the same period of storage under ambient conditions. Color changes on sealed devices could still be observed three weeks after fabrication, while unsealed ones produced significant less response after a week, and no color change was observed after three weeks of storage (Figure $2 \mathrm{~B}$ ). With polymer film protection, the lifetime of devices made with the porous paper substrate was greatly extended. 
(A)

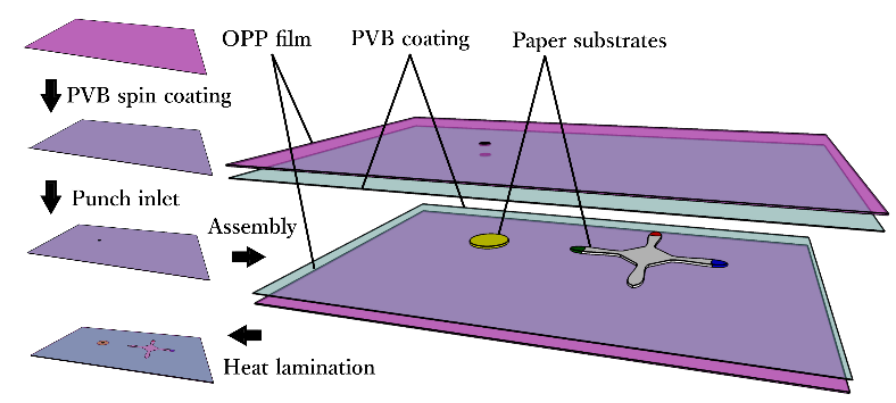

(B)
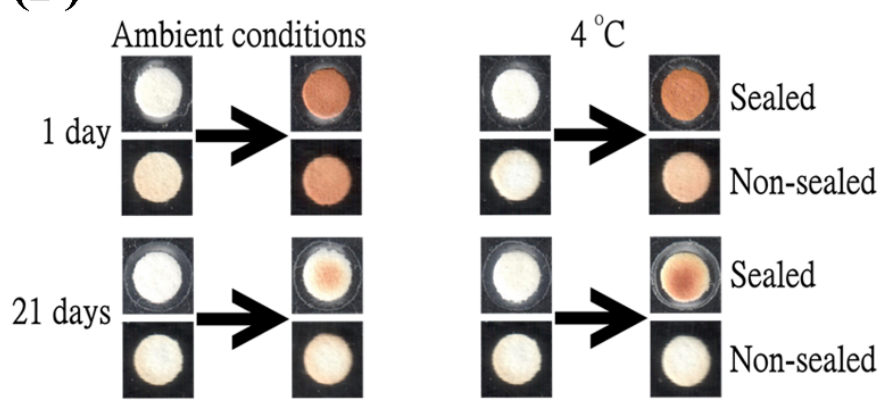

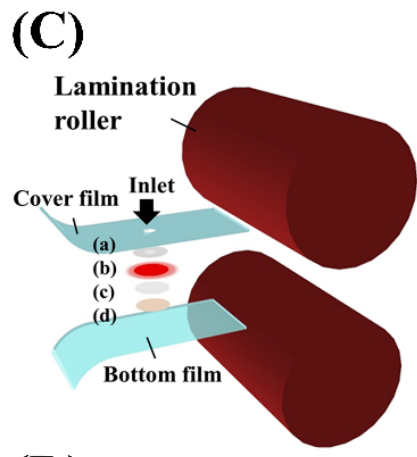

(D)

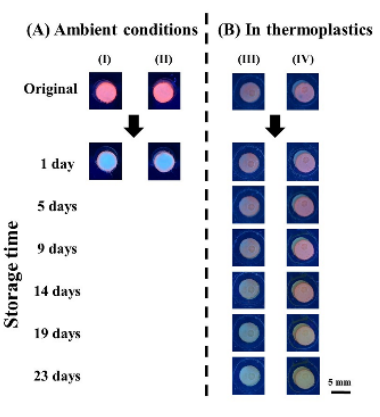

Figure 2. (A) Fabrication process of a paper-based device laminated with polypropylene/polyvinyl butyral (OPP/PVB) films; (B) reagents protected with OPP/PVB films exhibit extended device lifetime to 3 weeks. (C) Fabrication process of a paper-based device laminated with polyethylene terephthalate (PET) films while containing an extra antioxidative sheet; (D) reagents protected with PET films and a piece of antioxidative sheet exhibit extended lifetime to 23 days. Adapted with permission from $[14,16]$.

In other research, the reactivity of oxygen-sensitive copper nanoclusters on paper substrate was maintained for 23 days with assistance from polyethylene terephthalate (PET) film lamination and ascorbic acid-containing antioxidative sheet [9]. The red fluorescenceemitting copper nanocluster can be used to detect cysteine molecules when introduced onto the porous paper substrate. However, these nanoclusters are sensitive to oxygen and the fluorescent property is lost if this copper nanocluster-paper composite is stored under ambient conditions for just one day. Importantly, the device lifetime can be extended to five days with the help of enclosing the substrate in PET films, as material degradation is avoided via the prevention of oxygen transmission through PET films. A much longer device lifetime of 23 days was also achieved through the incorporation of antioxidative sheets. The additionally introduced ascorbic acid containing antioxidative paper sheets were able to react with the penetrated oxygen, resulting in prolonged device storage time.

As can be seen in these examples, the enrollment of multiple materials on a sensing device generates functional composites that can help to integrate practical platforms with more potent properties. It should also be noted that the introduction of additional components inside an analytical device has to follow the original needs to avoid undesirable issues.

\section{Signal Readout Enhancement}

From the device signal collection point of view, the use of composite materials for sensing device designs may lead to specific tasks that have to be solved in the route. As discussed in the last section, supplemental protection of paper substrates provides immense benefits in terms of device handling and reagent perseverance. Unfortunately, this extra layer of material coverage onto the main sensing substrates may result in unprecedented signal disturbance issues. For example, the commercially available PET lamination film exhibits a strong blue emission under ultraviolet excitation. This phenomenon induces 
troublesome background interferences if fluorescent reagents are used in conjunction with it. To circumvent this type of problem, a homemade lamination film prepared by spin coating polyvinyl butyral (PVB) on polypropylene (OPP) was invented by Wang et al. [7]. This composite film is free of blue emission under ultraviolet irradiation, and is compatible with the calcein blue-containing paper device for various metal ion fluorescent detection. By analyzing the grayscale intensity of photo images, devices laminated with OPP/PVB films not only yielded a lower limit of detection $(1.28 \mu \mathrm{M}$, compared to $10.45 \mu \mathrm{M}$ from devices with commercial PET film lamination), but a higher sensitivity was also obtained (as evident from the higher slope value) (Figure 3A). In addition, this material also functions normally under different $\mathrm{pH}$ values and supports multiplexed detection. These properties make this simple combination of polymers a suitable replacement for commercially available PET lamination films, especially when the use of fluorescent reagents is required.

(A)

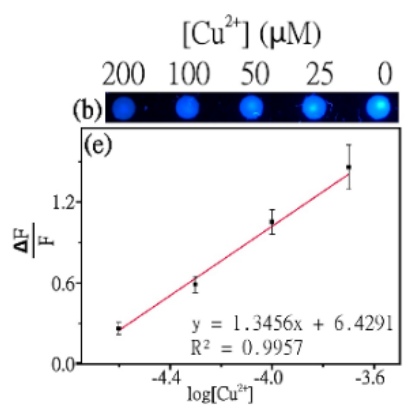

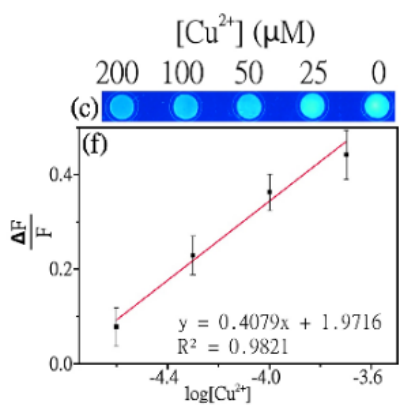

(B)

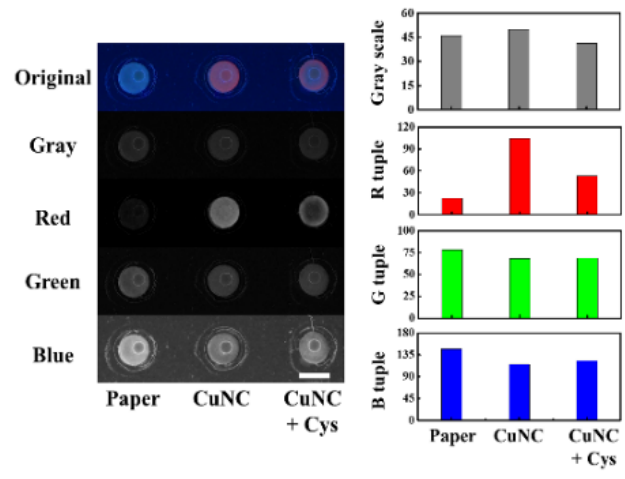

Figure 3. (A) The reduced blue fluorescence background from devices made with OPP/PVB lamination films (left) compared to devices made with PET films (right). (B) Enhanced device responses from monitoring only the red tuple of images collected from devices utilizing red fluorescence-emitting copper nanoclusters. Adapted with permission from [14,16].

Other than preparing low-background composite backing materials for signal improvements, the readout obstacle can also be mitigated by inventing new reagents and adopting new signal processing methods. A filter-free fluorescence detection of cysteine on a laminated paper-based device was designed by Chen et al. [9]. The system features red emitting copper nanoclusters to eliminate the fluorescent background signal without the need of optical filters. Since the copper nanocluster presents emission in the red color region, blue and green fluorescence interferences can be ignored during the photo image analysis step by monitoring the emission changes in the red tuple only (Figure 3B). The red fluorescence emission is quenched upon the addition of cysteine target molecules, and the blue fluorescence background from the paper substrate and the backing polymer film is therefore avoided under this operation. Owing to the pronounced fluorescence signal alteration in the red tuple, a greatly increased signal-to-background ratio ( $>4$ times) can be obtained by observing the red tuple as compared to other color tuples. In addition, a linear calibration curve with coefficient of determination $\left(R^{2}\right)$ over 0.99 can be obtained, while calibration curves collected from the grayscale image or other color tuples give values lower than 0.50 . The calibration sensitivity, limit of detection, and coefficient of determination are on par with those obtained from instrumental fluorescence analysis, indicating the effectiveness of this integrating approach for an optical filter-free paper-based sensing device.

Evidenced for the aforementioned examples, the signal readout of an analytical device based on porous composite materials can be improved to achieve a better sensing performance. This can not only be achieved through a proper selection of utilized materials, but can also be accomplished via a compatible integration of other useful techniques. 


\section{Beyond Chemical Sensors and Diversified Material Selections}

Porous materials are easily integrated with various solution-based reagents as the pores can store active ingredients, forming sensing devices based on chemical reactions. However, this concept is not limited within the chemical sensing category and can be extended into different research fields. For example, mechanical sensors can also be constructed by utilizing different aspects of the porous material for other intriguing purposes. By integrating with conductive additives, e.g., conductive polymers [17,39], metal nanoparticles [17], or carbon nanotubes [21,40,41], soft porous materials can become electrically conductive. This material property modification enriches the usefulness of produced composites, where a sensing device functioning from a different aspect can now be designed. When an external pressure stimulus is applied to the aforementioned composite material, contact point increases within the platform results in rising conductivity, which can be used to calculate the applied force. Pressure sensing devices based on this mechanism are termed piezoresistive sensors, and deliver a variety of useful recent applications, such as breath detection, pulse mentioning, and human-machine interfacing. The low-power consumption, straightforward sensor setup, and simple fabrication process led them to be more extensively studied compared to other types of pressure sensors. Conductive porous substrates, the core element in piezoresistive sensors, are often made by pairing a porous spongelike material, e.g., polyurethane [21,42,43], melamine [22,23], and polydimethylsiloxane [40,44], with conductive additives, e.g., poly(3,4-ethylenedioxythiophene):poly(styrene sulfonate) (PEDOT:PSS) and carbon nanotubes, in which the conductive additives are physically adsorbed on the substrate filaments.

Alternatively, cellulose-based paper material can also be used as the sensing device supporting substrate. A pressure sensor made by holding pieces of stacked conductive paper with lamination films was designed by Tsai et al. [17]. Filter paper fibers were first coated with the conductive polymer PEDOT, and their surfaces were thereafter decorated with silver nanoparticles (AgNPs). As a porous substrate, the filter paper piece provides sufficient space for force-induced deformation, while the PEDOT-coated fibers contribute to electrical conductivity. The in situ reduction of AgNPs onto PEDOT-coated fibers further increases overall conductivity, and enriches fiber surface roughness. By stacking multiple layers of AgNP-PEDOT-paper pieces and lamination into polypropylene films with copper wires for external circuit contacts, a composite material-based piezoresistive pressure sensor is produced (Figure 4A). The lamination process holds the composite material together to provide improved handling and robust pressure detection. Pressure sensors made with this design exhibit broad detection range (0-12 $\mathrm{kPa})$, high sensitivity $\left(0.119 \mathrm{kPa}^{-1}\right)$, and superior durability (stable over 2000 pressure cycles), which can be used to effectively detect various types of human motions.

In addition to exploring different composites of porous substrates and seeking conductivity modifications, details in device design can also be fine-tuned to improve the performance of piezoresistive pressure sensors. For example, alternate designs for the interface between the porous composite material and the external circuit can be adapted to greatly enhance the sensitivity of a piezoresistive pressure sensor [45]. Conventionally, silver paste is used at this interface to provide a thorough connection between the composite bulk sponge material and the external circuit. However, by replacing the silver paste with ordered metal microwires, the sensor's sensitivity is enhanced up to $10^{6}$-fold (Figure 4B). Prior to the application of pressure, the microwires are the only contact between the composite sponge material and the external circuit, leading to a high initial electrical resistance. When an external force is applied, the soft conductive substrate deforms and gradually collapses into the gaps between the microwires, resulting in increased contact area and decreased resistance. Accordingly, a large difference in resistance even under minute pressure can be used to construct a highly sensitive piezoresistive pressure sensor. This design functions regardless of the composite substrate's intrinsic property, which was proven by altering the base porous material and conductive modifications separately. 
(A)

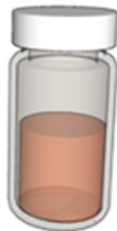

EDOT Polymerization

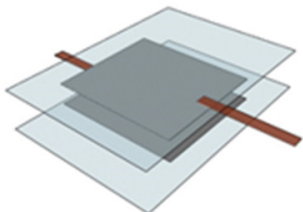

Device Assembly

(B)

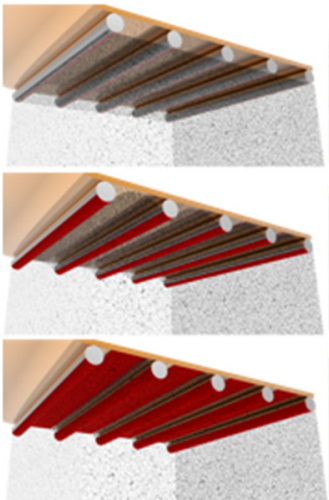

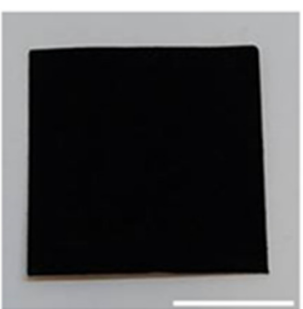
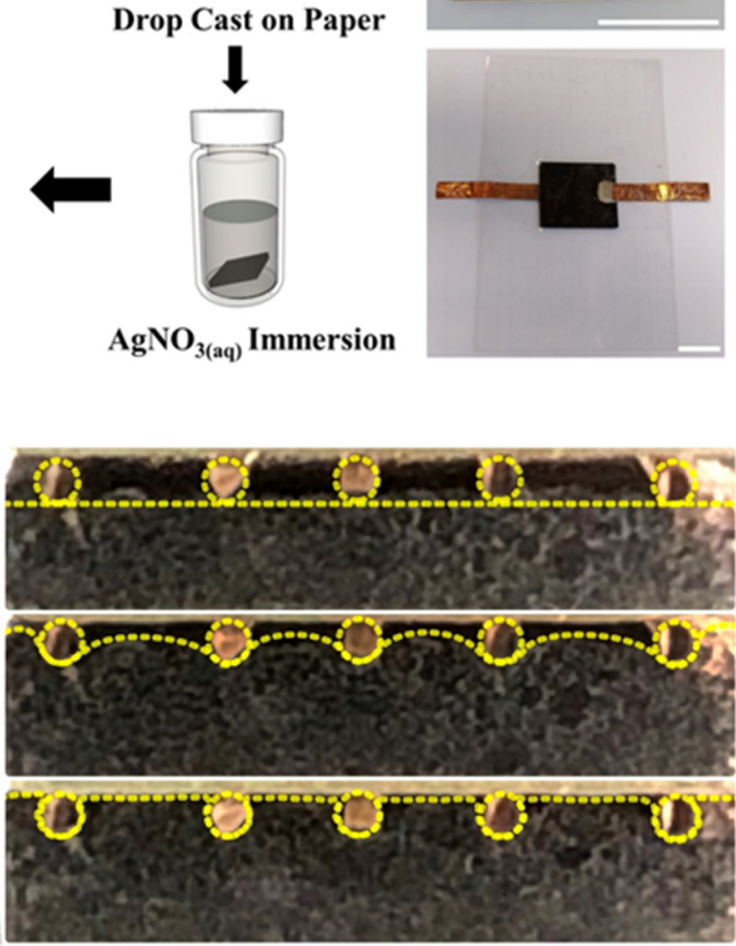

Figure 4. (A) Fabrication process of a piezoresistive pressure sensor made by stacking multiple pieces of silver nanoparticles-poly(3,4-ethylenedioxythiophene) (AgNP-PEDOT)-paper composite material. (B) An improved bulk material-electrode interface design for a sponge-based piezoresistive sensor. Adapted with permission from [27,37].

As can be learned from these examples, the use of porous composite materials should not be restricted within the chemical sensor study, but can be applied to various fields. This can be achieved by selecting suitable material pairs, and the device design is widely changeable based on researcher's interests.

\section{Future Perspective}

The potential of using porous composite materials to fabricate portable analytical devices is immense, which is attributed to its extremely high versatility. As a base material, the porous substrate can be arbitrarily patterned to render a hydrophilic or hydrophobic characteristic. It can then be further processed, allowing the design of a complex system even on a three-dimensional level. This advantageous property enables diverse opportunities, especially on designing a practical sensing device for specialized needs. Aside from examples given here, a myriad of useful designs has also been proposed. For example, origami devices incorporate multiplexed analysis within a compact platform by folding the patterned substrate. Meanwhile, channel enclosure devices can isolate the porous support from the environment through delicate partial modification of the substrate. Considering available detection methods on these devices, the material's porous nature makes it compatible with a wide array of common reagents, ranging from simple molecules and enzyme combinations to custom-made novel nanomaterials. Compatible signal output methods 
can therefore include colorimetric, fluorometric, and electrochemical detections, but are not limited to these. Furthermore, the assembled sensing device can be polished via the addition of protective layers to provide benefits regarding the mechanical strength and durability of the platform. Interestingly, in addition to chemistry-based sensing techniques, porous materials with proper composition and design can also become core elements in a different category of sensors, such as those described in numerous recently published studies on the topic of piezoresistive pressure sensing.

Despite the efforts devoted in this field, commercialized sensing devices utilizing porous composite materials for public analytical demands are still lacking. Nevertheless, various testing strips, e.g., pregnancy test strips and urine test strips, are the most prevalent ones on the market. These successful products are all simple and foolproof to operate, and often require no sample pretreatments. Besides, a straightforward and distinct binary signal readout that can easily be identified is also a guideline for researchers on designing practical sensing devices. Although novel tools and methods for sensing device fabrication can be found in abundance in literature, the technology readiness level of these published designs mostly remains at 4 to 6 , indicating that they are highly limited to demonstrations in laboratories, while some have been tested in real-world environments. The transition of these ideas into commercial products depends on the identification of market needs followed by appropriate available tools and components assembling. Overall, the fabrication of a sensing device based on porous composites depends on choosing and integrating the suitable components from the large library available, but more attention should be directed toward fitting designed devices to practical demands. We look forward to more exciting composite devices designed by scientists and engineers, which will be updated in the near future as more research is published.

Author Contributions: Conceptualization, writing, review, and editing were done by C.-M.W. and W.-S.L. All authors have read and agreed to the published version of the manuscript.

Funding: This research was funded by the Taiwan Ministry of Science and Technology, MOST 108-2628-M-002-011-MY3.

Conflicts of Interest: The authors declare no conflict of interest.

\section{References}

1. Noviana, E.; Carrao, D.B.; Pratiwi, R.; Henry, C.S. Emerging Applications of Paper-Based Analytical Devices for Drug Analysis: A Review. Anal. Chim. Chim. Acta 2020, 1116, 70-90. [CrossRef] [PubMed]

2. Boobphahom, S.; Ly, M.N.; Soum, V.; Pyun, N.; Kwon, O.S.; Rodthongkum, N.; Shin, K. Recent Advances in Microfluidic Paper-Based Analytical Devices toward High-Throughput Screening. Molecules 2020, 25, 2970. [CrossRef] [PubMed]

3. Wang, C.M.; Chen, C.Y.; Liao, W.S. Enclosed Paper-Based Analytical Devices: Concept, Variety, and Outlook. Anal. Chim. Acta 2021, 1144, 158-174. [CrossRef]

4. Reboud, J.; Xu, G.L.; Garrett, A.; Adriko, M.; Yang, Z.G.; Tukahebwa, E.M.; Rowell, C.; Cooper, J.M. Paper-Based Microfluidics for DNA Diagnostics of Malaria in Low Resource Underserved Rural Communities. Proc. Natl. Acad. Sci. USA 2019, 116, $4834-4842$. [CrossRef] [PubMed]

5. Hiraoka, R.; Kuwahara, K.; Wen, Y.C.; Yen, T.H.; Hiruta, Y.; Cheng, C.M.; Citterio, D. Paper-Based Device for Naked Eye Urinary Albumin/Creatinine Ratio Evaluation. ACS Sens. 2020, 5, 1110-1118. [CrossRef]

6. Mettakoonpitak, J.; Volckens, J.; Henry, C.S. Janus Electrochemical Paper-Based Analytical Devices for Metals Detection in Aerosol Samples. Anal. Chem. 2020, 92, 1439-1446. [CrossRef]

7. Wang, C.M.; Chen, C.Y.; Liao, W.S. Paper-Polymer Composite Devices with Minimal Fluorescence Background. Anal. Chim. Acta 2017, 963, 93-98. [CrossRef]

8. da Silva, V.A.O.P.; de Freitas, R.C.; de Oliveira, P.R.; Moreira, R.C.; Marcolino, L.H.; Bergamini, M.F.; Coltro, W.K.T.; Janegitz, B.C. Microfluidic Paper-Based Device Integrated with Smartphone for Point-of-Use Colorimetric Monitoring of Water Quality Index. Measurement 2020, 164, 108085. [CrossRef]

9. Chen, C.Y.; Chen, C.L.; Wang, C.M.; Liao, W.S. Laminated Copper Nanocluster Incorporated Antioxidative Paper Device with RGB System-Assisted Signal Improvement. Nanomaterials 2018, 8, 97. [CrossRef]

10. Wang, C.M.; Hsieh, C.H.; Chen, C.Y.; Liao, W.S. Low-Voltage Driven Portable Paper Bipolar Electrode-Supported Electrochemical Sensing Device. Anal. Chim. Acta 2018, 1015, 1-7. [CrossRef]

11. Ding, J.W.; Li, B.W.; Chen, L.X.; Qin, W. A Three-Dimensional Origami Paper-Based Device for Potentiometric Biosensing. Angez. Chem. Int. Ed. 2016, 55, 13033-13037. [CrossRef] [PubMed] 
12. Pungjunun, K.; Chaiyo, S.; Praphairaksit, N.; Siangproh, W.; Ortner, A.; Kalcher, K.; Chailapakul, O.; Mehmeti, E. Electrochemical Detection of NOx Gas based on Disposable Paper-Based Analytical Device using a Copper Nanoparticles-Modified Screen-Printed Graphene Electrode. Biosens. Bioelectron. 2019, 143, 111606. [CrossRef] [PubMed]

13. Cunningham, J.C.; Scida, K.; Kogan, M.R.; Wang, B.; Ellington, A.D.; Crooks, R.M. Paper Diagnostic Device for Quantitative Electrochemical Detection of Ricin at Picomolar Levels. Lab Chip 2015, 15, 3707-3715. [CrossRef] [PubMed]

14. Li, X.; Scida, K.; Crooks, R.M. Detection of Hepatitis B Virus DNA with a Paper Electrochemical Sensor. Anal. Chem. 2015, 87, 9009-9015. [CrossRef] [PubMed]

15. Wu, L.D.; Ma, C.; Ge, L.; Kong, Q.K.; Yan, M.; Ge, S.G.; Yu, J.H. Paper-Based Electrochemiluminescence Origami Cyto-Device for Multiple Cancer Cells Detection using Porous AuPd Alloy as Catalytically Promoted Nanolabels. Biosens. Bioelectron. 2015, 63, 450-457. [CrossRef] [PubMed]

16. Sun, Y.T.; Chang, Y.Y.; Zhang, Q.; Liu, M. An Origami Paper-Based Device Printed with DNAzyme-Containing DNA Superstructures for Escherichia coli Detection. Micromachines 2019, 10, 531. [CrossRef]

17. Tsai, Y.J.; Wang, C.M.; Chang, T.S.; Sutradhar, S.; Chang, C.W.; Chen, C.Y.; Hsieh, C.H.; Liao, W.S. Multilayered Ag NP-PEDOTPaper Composite Device for Human-Machine Interfacing. ACS Appl. Mater. Inter. 2019, 11, 10380-10388. [CrossRef]

18. Guo, S.X.; Zhang, C.H.; Yang, M.; Zhou, Y.L.; Bi, C.L.; Lv, Q.T.; Ma, N. A Facile and Sensitive Electrochemical Sensor for Non-Enzymatic Glucose Detection based on Three-Dimensional Flexible Polyurethane Sponge Decorated with Nickel Hydroxide. Anal. Chim. Acta 2020, 1109, 130-139. [CrossRef]

19. Guo, J.H.; Bai, Z.W.; Lyu, Y.L.; Wang, J.K.; Wang, Q. A Dual Ammonia-Responsive Sponge Sensor: Preparation, Transition Mechanism and Sensitivity. Analyst 2018, 143, 3390-3398. [CrossRef]

20. Zhang, C.; Hou, Z.L.; Zhang, B.X.; Fang, H.M.; Bi, S. High Sensitivity Self-Recovery Ethanol Sensor based on Polyporous Graphene Oxide/melamine Composites. Carbon 2018, 137, 467-474. [CrossRef]

21. Tewari, A.; Gandla, S.; Bohm, S.; McNeill, C.R.; Gupta, D. Highly Exfoliated MWNT-rGO Ink-Wrapped Polyurethane Foam for Piezoresistive Pressure Sensor Applications. ACS Appl. Mater. Interfaces 2018, 10, 5185-5195. [CrossRef]

22. Xu, R.X.; Zhang, K.L.; Xu, X.Y.; He, M.H.; Lu, F.C.; Su, B. Superhydrophobic WS2-Nanosheet-Wrapped Sponges for Underwater Detection of Tiny Vibration. Adv. Sci. 2018, 5, 1700655. [CrossRef] [PubMed]

23. Fang, X.L.; Tan, J.P.; Gao, Y.; Lu, Y.F.; Xuan, F.Z. High-Performance Wearable Strain Sensors based on Fragmented Carbonized Melamine Sponges for Human Motion Detection. Nanoscale 2017, 9, 17948-17956. [CrossRef] [PubMed]

24. Martinez, A.W.; Phillips, S.T.; Butte, M.J.; Whitesides, G.M. Patterned Paper as a Platform for Inexpensive, Low-Volume, Portable Bioassays. Angew. Chem. Int. Ed. 2007, 46, 1318-1320. [CrossRef]

25. Fenton, E.M.; Mascarenas, M.R.; Lopez, G.P.; Sibbett, S.S. Multiplex Lateral-Flow Test Strips Fabricated by Two-Dimensional Shaping. ACS Appl. Mater. Inter. 2009, 1, 124-129. [CrossRef]

26. Shibata, H.; Henares, T.G.; Yamada, K.; Suzuki, K.; Citterio, D. Implementation of a Plasticized PVC-Based Cation-Selective Optode System into a Paper-Based Analytical Device for Colorimetric Sodium Detection. Analyst 2018, 143, 678-686. [CrossRef]

27. Chen, C.Y.; Tan, Y.Z.; Hsieh, P.H.; Wang, C.M.; Shibata, H.; Maejima, K.; Wang, T.Y.; Hiruta, Y.; Citterio, D.; Liao, W.S. Metal-Free Colorimetric Detection of Pyrophosphate Ions by Inhibitive Nanozymatic Carbon Dots. ACS Sens. 2020, 5, 1314-1324. [CrossRef]

28. Carrilho, E.; Martinez, A.W.; Whitesides, G.M. Understanding Wax Printing: A Simple Micropatterning Process for Paper-Based Microfluidics. Anal. Chem. 2009, 81, 7091-7095. [CrossRef]

29. Bruzewicz, D.A.; Reches, M.; Whitesides, G.M. Low-Cost Printing of Poly(dimethylsiloxane) Barriers to Define Microchannels in Paper. Anal. Chem. 2008, 80, 3387-3392. [CrossRef]

30. Liu, H.; Crooks, R.M. Three-Dimensional Paper Microfluidic Devices Assembled Using the Principles of Origami. J. Am. Chem. Soc. 2011, 133, 17564-17566. [CrossRef]

31. Thom, N.K.; Lewis, G.G.; Yeung, K.; Phillips, S.T. Quantitative Fluorescence Assays using a Self-Powered Paper-Based Microfluidic Device and a Camera-Equipped Cellular Phone. RSC Adv. 2014, 4, 1334-1340. [CrossRef] [PubMed]

32. Uzek, R.; Sari, E.; Senel, S.; Denizli, A.; Merkoci, A. A Nitrocellulose Paper Strip for Fluorometric Determination of Bisphenol A using Molecularly Imprinted Nanoparticles. Microchim. Acta 2019, 186, 218. [CrossRef] [PubMed]

33. Boobphahom, S.; Ruecha, N.; Rodthongkum, N.; Chailapakul, O.; Remcho, V.T. A Copper Oxide-Ionic Liquid/Reduced Graphene Oxide Composite Sensor Enabled by Digital Dispensing: Non-Enzymatic Paper-Based Microfluidic Determination of Creatinine in Human Blood Serum. Anal. Chim. Acta 2019, 1083, 110-118. [CrossRef] [PubMed]

34. Mettakoonpitak, J.; Boehle, K.; Nantaphol, S.; Teengam, P.; Adkins, J.A.; Srisa-Art, M.; Henry, C.S. Electrochemistry on Paper-based Analytical Devices: A Review. Electroanalysis 2016, 28, 1420-1436. [CrossRef]

35. Adkins, J.; Boehle, K.; Henry, C. Electrochemical Paper-Based Microfluidic Devices. Electrophoresis 2015, 36, 1811-1824. [CrossRef]

36. Cassano, C.L.; Fan, Z.H. Laminated Paper-Based Analytical Devices (LPAD): Fabrication, Characterization, and Assays. Microfluid. Nanofluid. 2013, 15, 173-181. [CrossRef]

37. Liu, W.; Cassano, C.L.; Xu, X.; Fan, Z.H. Laminated Paper-Based Analytical Devices (LPAD) with Origami-Enabled Chemiluminescence Immunoassay for Cotinine Detection in Mouse Serum. Anal. Chem. 2013, 85, 10270-10276. [CrossRef]

38. de Oliveira, R.A.G.; Camargo, F.; Pesquero, N.C.; Faria, R.C. A Simple Method to Produce 2D and 3D Microfluidic Paper-Based Analytical Devices for Clinical Analysis. Anal. Chim. Acta 2017, 957, 40-46. [CrossRef] 
39. Ding, Y.C.; Yang, J.; Tolle, C.R.; Zhu, Z.T. Flexible and Compressible PEDOT:PSS@Melamine Conductive Sponge Prepared via One-Step Dip Coating as Piezoresistive Pressure Sensor for Human Motion Detection. ACS Appl. Mater. Inter. 2018, 10, 16077-16086. [CrossRef]

40. Rinaldi, A.; Tamburrano, A.; Fortunato, M.; Sarto, M.S. A Flexible and Highly Sensitive Pressure Sensor Based on a PDMS Foam Coated with Graphene Nanoplatelets. Sensors 2016, 16, 2148. [CrossRef]

41. Song, Y.; Chen, H.T.; Su, Z.M.; Chen, X.X.; Miao, L.M.; Zhang, J.X.; Cheng, X.L.; Zhang, H.X. Highly Compressible Integrated Supercapacitor-Piezoresistance-Sensor System with CNT-PDMS Sponge for Health Monitoring. Small 2017, 13, 1702091. [CrossRef] [PubMed]

42. Yao, H.B.; Ge, J.; Wang, C.F.; Wang, X.; Hu, W.; Zheng, Z.J.; Ni, Y.; Yu, S.H. A Flexible and Highly Pressure-Sensitive GraphenePolyurethane Sponge Based on Fractured Microstructure Design. Adv. Mater. 2013, 25, 6692-6698. [CrossRef] [PubMed]

43. Ma, Z.L.; Wei, A.J.; Ma, J.Z.; Shao, L.; Jiang, H.E.; Dong, D.D.; Ji, Z.Y.; Wang, Q.; Kang, S.L. Lightweight, Compressible and Electrically Conductive Polyurethane Sponges Coated with Synergistic Multiwalled Carbon Nanotubes and Graphene for Piezoresistive Sensors. Nanoscale 2018, 10, 7116-7126. [CrossRef]

44. Han, J.W.; Kim, B.; Li, J.; Meyyappan, M. Flexible, Compressible, Hydrophobic, Floatable, and Conductive Carbon NanotubePolymer Sponge. Appl. Phys. Lett. 2013, 102, 051903. [CrossRef]

45. Wang, C.M.; Hsieh, C.H.; Liao, W.S. Enhancing Piezoresistive Pressure Response Device Sensitivity by Orders of Magnitude. Adv. Mater. Interfaces 2020, 7, 1902202. [CrossRef] 